\title{
Successful Encoding during Natural Reading Is Associated with Fixation-Related Potentials and Large-Scale Network Deactivation
}

\author{
[D Naoyuki Sato, ${ }^{1}$ and ${ }^{1}$ Hiroaki Mizuhara ${ }^{2}$
}

https://doi.org/10.1523/ENEURO.0122-18.2018

${ }^{1}$ Department of Complex and Intelligent Systems, School of Systems Information Science, Future University Hakodate, Hokkaido 041-8633, Japan, ${ }^{2}$ Graduate School of Informatics, Kyoto University, Sakyo-ku, Kyoto 606-8501, Japan

\begin{abstract}
Reading literature (e.g., an entire book) is an enriching experience that qualitatively differs from reading a single sentence; however, the brain dynamics of such context-dependent memory remains unclear. This study aimed to elucidate mnemonic neural dynamics during natural reading of literature by performing electroencephalogram (EEG) and functional magnetic resonance imaging (fMRI). Brain activities of human participants recruited on campus were correlated with their subsequent memory, which was quantified by semantic correlation between the read text and reports subsequently written by them based on state of the art natural language processing procedures. The results of the EEG data analysis showed a significant positive relationship between subsequent memory and fixation-related EEG. Sentence-length and paragraph-length mnemonic processes were associated with N1-P2 and P3 fixation-related potential (FRP) components and fixation-related $\theta$-band $(4-8 \mathrm{~Hz})$ EEG power, respectively. In contrast, the results of fMRI analysis showed a significant negative relationship between subsequent memory and blood oxygenation level-dependent (BOLD) activation. Sentence-length and paragraph-length mnemonic processes were associated with networks of regions forming part of the salience network and the default mode network (DMN), respectively. Taken together with the EEG results, these memory-related deactivations in the salience network and the DMN were thought to reflect the reading of sentences characterized by low mnemonic load and the suppression of task-irreverent thoughts, respectively. It was suggested that the context-dependent mnemonic process during literature reading requires large-scale network deactivation, which might reflect coordination of a range of voluntary processes during reading.
\end{abstract}

Key words: memory encoding; natural reading; neural oscillation

\section{Significance Statement}

Context-dependent memory encoding during natural reading of literature was evaluated using electroencephalography (EEG) and functional magnetic resonance imaging (fMRI) based on a subsequent memory paradigm. Subsequent memory was quantified by semantic correlation between the read text and reports subsequently written by the participants, based on a recent natural language processing procedure. Our results demonstrated a positive correlation between subsequent memory and fixationrelated EEG and a negative correlation with fMRI activity. Sentence-length and paragraph-length processes were associated with regions belonging to the salience network and the default mode network, respectively. This is the first demonstration that memory encoding during literature reading is associated with large-scale network deactivations, which might reflect the coordination of a range of voluntary processes during reading. 


\section{Introduction}

Neural dynamics during memory encoding have been extensively investigated using the subsequent memory paradigm (Brewer, 1998; Wagner, 1998), in which brain activity during encoding of items that are subsequently remembered is compared to the activity during encoding of items that are subsequently forgotten. Functional magnetic resonance imaging (fMRI) studies have demonstrated that multiple brain regions, including the inferior frontal cortex and hippocampus, are activated during successful memory encoding of words (Wagner, 1998), pictures (Brewer, 1998), and item-context associations (Summerfield and Mangels, 2005; for review, see Kim, 2011). This activation is termed the subsequent memory effect (SME). In contrast, the activation of distinct brain regions, such as the anterior and posterior midline cortices, was also observed during unsuccessful encoding; an effect termed the "subsequently forgotten effect" or "negative subsequent memory effect" (NSME; Otten and Rugg, 2001; Wagner and Davachi, 2001; Daselaar et al., 2004).

Electroencephalography (EEG) studies found that specific event-related potentials (ERPs) and brain oscillations occur during the SME. Specifically, the P3 ERP component increases during successful encoding of words (Klimesch et al., 2000). EEG $\theta(4-7 \mathrm{~Hz})$ oscillations increased during successful encoding of words (Klimesch et al., 1996), pictures (Osipova et al., 2006), and item-context binding (Summerfield and Mangels, 2005; for review, see Nyhus and Curran, 2010) . However, opposite effects, namely EEG $\theta$ decreases during successful encoding, have also been reported, suggesting that the SME primarily reflects perceptual and cognitive processes engaged by the encoding tasks (Hanslmayr and Staudigl, 2014). In these studies, relatively simple memory contents, such as single words or itemlocation pairs have been used. Thus, it is of interest to investigate whether the same neural dynamics is relevant for the encoding of a more natural form of memory consisting of semantically richer and context-dependent material, for example the experience of reading literature.

Literature reading provides a good example of a semantically rich and context-dependent experience for evaluating natural memory. The neural dynamics of reading has been

Received March 29, 2018; accepted September 23, 2018; First published October 22, 2018

The authors declare no competing financial interests.

Author contributions: N.S. and H.M. designed research; N.S. and H.M. performed research; N.S. analyzed data; N.S. wrote the paper.

This work was partially supported by the Japan Society for the Promotion of Science (JSPS) KAKENHI Grant 26540069 and The Ministry of Education,Culture,Sports,Science and Technology (MEXT) KAKENHI Grant $16 \mathrm{H} 01618$ (Nonlinear Neuro-oscillology).

Acknowledgements: This study was conducted using the MRI scanner and related facilities at Kokoro Research Center, Kyoto University.

Correspondence should be addressed to Naoyuki Sato, Department of Complex and Intelligent Systems, School of Systems Information Science, Future University Hakodate, 116-2 Kamedanakano, Hakodate, Hokkaido 0418655, Japan, E-mail: satonao@fun.ac.jp.

https://doi.org/10.1523/ENEURO.0122-18.2018

Copyright (C) 2018 Sato and Mizuhara

This is an open-access article distributed under the terms of the Creative Commons Attribution 4.0 International license, which permits unrestricted use, distribution and reproduction in any medium provided that the original work is properly attributed. extensively tested with sequential presentation of words constituting sentences, using EEG (Kutas and Hillyard, 1980; Kutas and Federmeier, 2000; Hagoort et al., 2004; Bastiaansen and Hagoort, 2006) and fMRI (Cutting et al., 2006; Pallier et al., 2011). Moreover, the neural dynamics of narrativelevel context have been evaluated by EEG and $\mathrm{AMRI}$ studies (Ferstl and von Cramon, 2001; Xu et al., 2005; Hasson et al., 2007; Yarkoni et al., 2008; Brennan, 2016). Some of these studies (Hasson et al., 2007; Yarkoni et al., 2008) demonstrated a subsequent memory effect during narrative comprehension. However, the examination of semantic contents after reading in these studies was based on a small number of questions regarding the text, and these were found to be too abstract for capturing the entire semantics of the text being read. Thus, the mnemonic process relevant to semantically rich content during reading needs further elucidation.

Recently developed natural-language techniques are expected to be useful for quantifying the semantic content of subsequent memory. One of these is "distributed semantic representation," in which each word is transformed to a vector consisting of intermediate semantic features. This technique is used to perform text comparisons based on semantic correlation rather than the appearance of particular keywords (Blei et al., 2003; Mikolov et al., 2013). The same technique has already been employed in $\mathrm{FMRI}$ studies showing that intermediate features were associated with widely distributed cortical regions (Mitchell et al., 2008; Huth et al., 2012). These studies are important in that they support the plausibility of using intermediate features in the investigation of brain activities related to semantics. Given the assumption that subsequent memory performance depends on a particular pattern of cortical activation during encoding into long-term memory, these same intermediate features may be used for the evaluation of subsequent memory of the texts.

In this study, we aimed to elucidate mnemonic neural dynamics during natural reading of literature using EEG and fMRI measurements. Brain activity was analyzed by comparing the measurements with content reports subsequently written by the participants based on a recently developed natural language technique quantifying semantic correlations to body of text. EEG measurement was combined with eye tracking to enable FRP analysis during reading, by which neural dynamics during free-viewing was assessed, while solving the problem of ocular artifact contamination in EEG signals (Dimigen et al., 2011; Henderson et al., 2013). We specifically asked the following questions: (1) What neural dynamics underlies memory encoding during natural reading of literature? (2) What neural dynamics is associated with multi-scaled contextual processing during reading of sentences and paragraphs?

\section{Materials and Methods}

We performed EEG and fMRI measurements separately during natural reading of identical texts. The task procedures and statistical analyses in these two experiments were designed to be comparable.

\section{EEG methods}

\section{Subjects}

Fifteen volunteers (two female, two left-handed, native Japanese speakers; 20-34 years old; mean \pm SD: $22.5 \pm$ 
3.5 years old) were recruited via poster advertisement at Future University Hakodate. They had no experience of neurologic disorders or use of psychotropic medications and had normal visual acuity, except that the eyes of six participants were corrected by spectacles and the eyes of one participant were corrected by intraocular lenses. They were compensated $\$ 25-30$ for participating in the study; the exact amount was prescribed by the city and depended on the school year of the participant in the Future University Hakodate. They provided written informed consent before participating in the experiment. The study was approved by the Ethics Committee of the Future University Hakodate. Data from the two left handed volunteers were ultimately excluded from the analysis.

\section{Stimuli}

Four scientific essays written by Torahiko Terada, entitled "Eagle's eye and olfaction," "Rhythms of poems," "Physiologic responses to seeing a movie," and "A case study of a ghost," were used as stimuli (Aozora Bunko, http://www.aozora.gr.jp/index_pages/person42.html ). They were selected as logical and non-emotional texts, suitable for high school students (unlike scientific articles). Each text was modified to modern kana (Japanese syllabary spelling system) with a length of $1919.5 \pm 77.6$ words (2973.3 \pm 137.3 characters, sentence length: 46.2 \pm 22.9 words, paragraph length: $116.2 \pm 82.4$ words; mean \pm SD). Their readability was measured as having a grade of "beyond high school (13)" using a program based on a statistical language model, Obi-2 (Sato et al., 2008).

During reading, the essays were presented on a 21 -inch monitor (Sony, CPD-G520), as a line of segmented text with 40 characters, displayed as $985 \times 24$ pixels (subtending $27.9 \times 0.74^{\circ}$ ) in white, on a black background. The single line presentation was used to restrict eye movements to horizontal saccades or eye blinks (Dimigen et al., 2011). The participants voluntarily advanced to the next line by pushing a button with the right thumb. Returning to a previous line was not allowed.

\section{Procedure}

Following the placement of EEG electrodes on the participants' heads, they read the four essays in a random order with rest intervals. During reading, the head position was stabilized by chin and forehead rests. At the beginning of each reading session, the eye movement system was calibrated with a nine-point grid. Following the reading session, the electrodes were removed, and the subjects washed their hair to remove conducting gel (which lasted for $\sim 15 \mathrm{~min}$ ). The subjects were then seated at a desk and wrote a summary of the content read (content report), as detailed as possible, following the order in which the essays were read. During this procedure, the interval between essay encoding and retrieval was $\sim 30$ min, and no explicit opportunities for rehearsing the essays were included. Possible influence of rehearsals during the interval was thought to be reduced by the separation of particular encoding and retrieval pairs using other essays tasks and the counter-balanced order of the text presentation. Before the main experiment, subjects performed a training session with a short essay (714 words) to familiarize themselves with the task.

\section{Eye movement data acquisition}

Eye movements were recorded binocularly with an infrared video-based eye tracker (EyeLink CL, SR Research Systems) at a sampling rate of $250 \mathrm{~Hz}$. During reading, saccades were detected by EyeLink software using an eye-movement velocity threshold of $30 \%$, an acceleration threshold of $8000 \% / \mathrm{s}^{2}$ and a saccadic threshold of $0.15^{\circ}$. Data from the right eye were analyzed, and those from the left eye were used only for validation. The following atypical fixations were discarded from the analysis: fixations separated vertically by 48 pixels (two characters) from the line, fixations with a duration of $<50 \mathrm{~ms}$ or $>750$ $\mathrm{ms}$, fixations at the first or the last saccades during the reading of each line, fixations for small ( $<1$ character) or large ( $>20$ character) saccades, and fixations shortly preceding/following an eye blink (ranging from -500 to 1000 ms of blink onset).

\section{EEG data acquisition and preprocessing}

EEG and EOG data were acquired using $\mathrm{Ag} / \mathrm{AgCl}$ electrodes with a BrainVision amplifier (BrainProducts). Twentyone electrodes were mounted on the scalp according to the standard 10-20 system without Fp1 and Fp2. Four EOG electrodes were affixed to the left and right outer eye canthi and above and below the right eye. EEG data (0.01- to $100-\mathrm{Hz}$ bandpass, $500 \mathrm{~Hz}$ sampling rate, impedance of the electrode $12.6 \pm 11.6 \mathrm{k} \Omega$; mean $\pm \mathrm{SD}$ ) were referenced to the $\mathrm{FCz}$ electrode during measurements and re-referenced to the average signal recorded at electrodes placed on the two earlobes for analysis.

Ocular artifacts were corrected by independent component analysis (Henderson et al., 2013). First, a dataset dominantly including ocular artifacts, given by fixationrelated data from -120 to $50 \mathrm{~ms}$ from the fixation onset $[20,443 \pm 11,008$ time points $(481.8 \pm 259.0$ trials; mean $\pm \mathrm{SD}) \times 26$ electrodes, with additional bandpass filtering between 1 and $50 \mathrm{~Hz}$ ], were collected and their independent components were calculated by FastICA (Hyvärinen and Oja, 2000). Second, independent components highly correlated to either horizontal, vertical, or radial EOGs (correlation coefficient of the entire time course across trials $>0.15$ ) were discarded, and the same separation matrix, calculated from the subset of the data, was applied to the original data. By this procedure, $5.5 \pm 2.3$ components (mean $\pm \mathrm{SD}$ ) were discarded; this rejection rate was similar to the rate reported previously (Henderson et al., 2013). Finally, the corrected EEG signals were filtered between 1 and $40 \mathrm{~Hz}$ (using a zero-lag Butterworth filter with $-12 \mathrm{~dB} /$ octave roll-off) and down-sampled to $250 \mathrm{~Hz}$ to match the eye-movement data. EEG and eye movement data were synchronized by a common trigger input from the stimulus computer.

\section{Text data analysis}

The analysis of the text data can be outlined as follows (Fig. 1A). Each text consisting of an arbitrary number of words was translated into a semantic vector consisting of intermediate semantic features (Blei et al., 2003; Mikolov et al., 2013). Statistically, natural texts consist of approxi- 
A

\section{Original text:}

Eagles can find a dead body of a mouse on the ground from high altitude. Visual angle of the mouse is ...

Semantic vector

\begin{tabular}{|c|c|c|c|c|c|}
\hline 0.20 & 0.01 & 0.10 & י & 0.00 & \\
\hline "flight" & "vision" & "science" & & "sports" & $\begin{array}{l}\text { Text } \\
\text { correlation }\end{array}$ \\
\hline 0.01 & 0.20 & 0.10 & . . & 0.00 & \\
\hline
\end{tabular}

\section{Content report:}

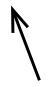

Vision of birds is sensitive to be able to catch a mouse on the ground....
B

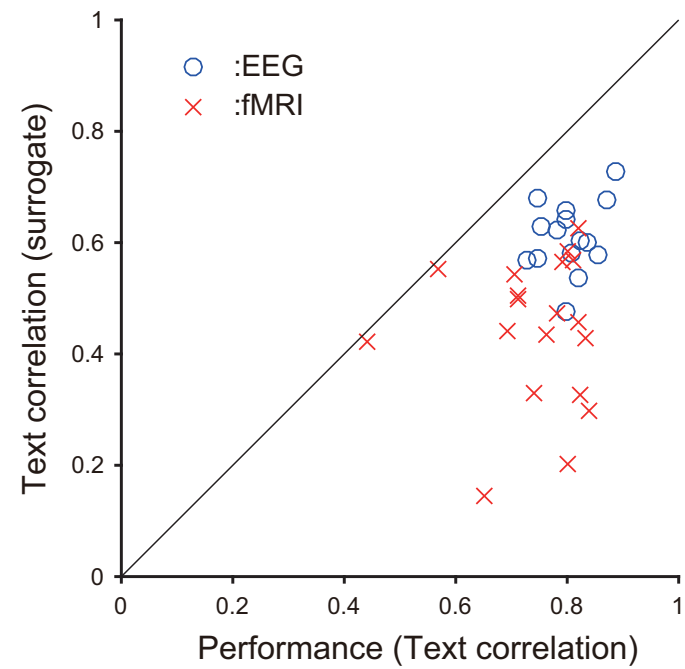

Figure 1. Computation of text correlations between the text that was read and the content report subsequently written by the participants. A, Schematic procedure for text comparison. Each text and content report were translated into a semantic vector consisting of intermediate semantic features computed from word-collocation in a large text database. The correlation of the vectors represents the semantic similarity between the two texts rather than the appearance of specific keywords (see text for details). $\boldsymbol{B}$, Performance of EEG and fMRI participants. Blue and red plots indicate the participants in the EEG and fMRI experiments, respectively. Horizontal and vertical axes denote the performance (the entire text correlation between the text read and the content report) and the surrogate text correlation, respectively. The fact that plots lie below the diagonal line shows that the content reports specifically reflected the texts read. The data from two participants, for whom the plots appeared along the diagonal line and whose correlations were below 0.6, were excluded from the analysis.

mately ten thousand types of words and the collocation matrix appears sparse. Therefore the relationship between the words (i.e., collocation matrix) can be computationally compressed and the dimension of the compressed word relationship typically falls within the range of several hundred dimensions (Landauer and Dumais, 1997). This compressed word relationship produces a word-to-vector map. When a text is represented by the average of the word vectors relevant to the words in the text ("bag-of-words"), the averaged vector, termed the "semantic vector," captures the intermediate semantics of the text, in a way that is robust against the influence of synonyms or rephrasing. For example, when two intermediate semantic features represent the amount of "flying" or "vision" (Fig. 1A), a semantic vector represents its semantics by the combined amounts of these semantic features, although in reality these semantic features were automatically produced by the above algorithms to optimally cover natural texts. The text that was read and the content reports subsequently written by the participants were individually translated into semantic vectors, and their correlation was used as an index for semantic text correlation evaluating the subsequent memory.

The detailed procedure applied in the text data analysis consisted of the following steps.

Step 1 The vector features of all words were computed from word-occurrence data within a large text corpus [Balanced Corpus of Contemporary Written Japanese (BCCWJ; Maekawa et al., 2014); Library/Book sub-corpus (10,551 texts, 60,615 word types, fixed length of 1000 characters per text) using the algorithm Word2Vec (Mikolov et al., 2013); using the parameters: context window of 10 words, CBOW model, and negative word sampling of 15 words)]. For this process we used open source code from https://code. google.com/archive/p/word2vec/.

Step 2 The words in every text were segmented into morphologic units ("short-unit word") using the Japanese morphologic analyzer MeCab (http://taku910.github.io/ mecab/) with the dictionary unidic-mecab (ver.2.1.3; https://ja.osdn.net/projects/unidic/).

Step 3 Each open-class word (noun, verb, adverb, and adjective) within the text was represented by a 100dimensional vector $\mathbf{w}_{i}$, where $i$ denotes word position in the given text.

Step 4 Three types of text correlation were calculated using sentence-length samples, paragraph-length samples, or the entire text. In the calculation, each text unit was represented by the average of the word vector, $\mathbf{T}_{\mathrm{T}}=1 /|\mathrm{T}| \sum_{i \in \mathrm{T}} \mathbf{w}_{i}$, where $\mathbf{w}_{i}$ is the $i$-th word vector in the text, and T is the set of word IDs in the text. The correlation of a pair of texts represented by the semantic vectors $\mathbf{T}_{E}$ and $\mathbf{T}_{R}$ was determined by cosine similarity, $C$ $\left(\mathbf{T}_{E}, \mathbf{T}_{R}\right)=\mathbf{T}_{E} \mathbf{T}_{R} /\left(\left|\mathbf{T}_{E}\right|\left|\mathbf{T}_{R}\right|\right)$, where the values range from $-1-1,0$ indicates an independent text pair, and 1 indicates an identical text pair.

Step 5 The correlation between the entire text read and the content report was termed the "performance" and used as a quality index for the individual content reports. To clarify whether the content reports specifically reflected the corresponding texts, surrogate text correlations, calculated as the average of correlations between the content report and non-corresponding texts, were 
additionally computed. The feature dimension (100) was determined to maximize the difference between the text read-content report correlation and the surrogate text correlations.

Step 6 In the following subsequent memory analysis, each sentence or paragraph of the text read was compared with the entire content report to identify which part of the text was reflected in the content report. The sentence-length correlations changed quickly as a function of word position, and paragraph-length correlations slowly because the former reflected the appearance of specific sentences while the latter reflected the appearance of abstract themes governing several sentences forming a paragraph. In the current analysis, text correlation was not highly sensitive to variation of word length in sentences or paragraph (i.e., results with fixed-length text correlations using the averaged sentence- or paragraphlength were fundamentally unchanged from the current results; data were partially shown by the author (Sato, 2015), thus the influence was not corrected.

\section{EEG data analysis}

The corrected EEG signals were analyzed in terms of FRPs and fixation-related time-frequency power as follows. First, the corrected EEG signals were segmented from -500 to $1000 \mathrm{~ms}$ from the fixation onset given by the eye movement data. Segments including data points within a limit of $\pm 80 \mu \mathrm{V}$ were used. Second, the influence of overlapped FRPs from neighboring fixations was reduced by subtracting an estimated FRP calculated from de-convolution by the use of the adjacent response technique (Woldorff, 1993). Third, fixation-related time-frequency EEG power was calculated using complex Morlet wavelet transformation (width $=5$ ), where 19 frequency bands were determined on a logarithmic scale $(2 \wedge(1,1.25, \ldots, 5.5) \mathrm{Hz})$ and split into four distinct bands, $4-7 \mathrm{~Hz}(\theta), 8-12 \mathrm{~Hz}(\alpha)$, $14-28 \mathrm{~Hz}(\beta)$, and $30-48 \mathrm{~Hz}(\gamma)$. The baseline for each power was subtracted; the baseline was calculated from the period within -300 to $-100 \mathrm{~ms}$ from the fixation onset. Finally, the time-frequency EEG power at time $t$ and frequency $f, P$ $(t, f)$, was compared with the text correlation between the part of the text read at eye fixation and the content report. Two text correlations, one at sentence-length and another at paragraph-length, $C_{S}$ and $C_{P}$, were used in the analysis. The time-frequency power was analyzed by multiple regression using the two text correlations, as (Eqn. 1):

$$
P(t, f)=b_{0}+b_{S} C_{S}^{\prime}+b_{P} C_{P}^{\prime},
$$

where $C_{S}^{\prime}$ and $C_{P}^{\prime}$ are the sentence-length and paragraphlength text correlations with a modification of GramSchmidt orthogonalization. The regression coefficients $b_{S}$ and $b_{P}$ were calculated separately for each electrode, each time point and each frequency band, and then integrated across all subjects using the $t$ statistic. Multiple comparisons in the $t$ tests were corrected using the nonparametric clustering permutation test (Maris and Oostenveld, 2007) with 4000 shuffled data sets, in which the statistical threshold was provided by a single procedure taking into account the electrodes, time, and frequency simultaneously. FRPs were also analyzed using the iden- tical regression analysis, where the regression coefficients were calculated separately for each electrode and each time point and then integrated across all subjects using the $t$ statistic. Additionally, to quantify the potential contribution of ocular artifact residuals in the corrected EEG, saccade size and fixation duration were analyzed using the same type of regression analysis.

\section{fMRI methods}

\section{Subjects}

Nineteen volunteers (10 female, all right-handed, native Japanese speakers; aged from 21 to 29 years old; mean \pm SD: $23.9 \pm 2.5$ years old) were recruited via poster advertisement at Kyoto University. They had no experience of neurologic disorders or use of psychotropic medications and had normal visual acuity, except that the eyes of 13 participants were corrected by spectacles. They were compensated $\$ 65$ for participating in the study. Written informed consent was provided before participating in the experiment. The experimental protocol was approved by the ethics committee at the Unit for Advanced Studies of the Human Mind, Graduate School of Medicine and the Faculty of Medicine, Kyoto University. Data from two volunteers were excluded from the analysis because of low memory performance.

\section{Procedure}

The procedure was as described above for the EEG experiment. The participants read the two essays at a natural pace within an MR scanner for $10 \mathrm{~min}$. The texts were two out of four essays used in the EEG experiment, the titles of which were "Eagle's eye and olfaction" and "Physiologic responses to seeing a movie." While reading, two lines of the text (60 characters) were displayed, with the participant voluntarily advancing to the next page by pressing a button (the essays consisted of 50 and 54 pages, respectively). To have page transition intervals longer than the timescale of hemodynamic response (for stable regressing out of the influence of page transition and button pressing in the following analysis), the number of characters in a page was changed to be larger than that of the EEG experiment. After the two texts were read, a 5 -min structural scan was performed. Following structural scanning, the participants sat at a desk outside the scanner and wrote two content reports within $15 \mathrm{~min}$, following the order in which the essays were read in as much detail as possible. In this procedure, the interval between essay encoding and essay retrieval was $\sim 20 \mathrm{~min}$.

\section{fMRI data acquisition and preprocessing}

During reading, blood oxygenation-sensitive echoplanar images (EPIs) were acquired using the 3T MR scanner (Magnetom Verio, Siemens) under the following conditions: repetition time $=2 \mathrm{~s}$, echo time $=30 \mathrm{~ms}$, flip angle $=80^{\circ}$, field of view $=192 \mathrm{~mm}$, in-plane resolution $=64 \times$ 64,30 axial slices, slice thickness $=5 \mathrm{~mm}$. One session lasted for $\leq 10 \mathrm{~min}$ (297 scans) as defined by the reading time for each essay. Two sessions were performed, one for each essay. After reading, a T1-weighted anatomic volume was acquired.

We used SPM8 software (Wellcome Department of Cognitive Neurology, London, United Kingdom; www.fil. 
ion.ucl.ac.uk/spm) for image preprocessing and voxelbased statistical analysis. The initial five scans in each session were discarded from the analysis to eliminate magnetic saturation effects. The remaining EPIs $(\leq 292$ scans $\times$ two sessions) were mapped to the first image volume for each participant to correct for head motion. The slice timing was corrected with respect to the middle slice to remove the time delay of scanning the entire brain. The individual EPIs were normalized to a standard brain by applying the parameters estimated by matching the T1 anatomic image to the stereotactic image in Montreal Neurologic Institute coordinates. The EPIs were then smoothed with an 8-mm full-width at half-maximum Gaussian kernel.

\section{fMRI analysis}

A voxel-based statistical analysis was performed on the preprocessed EPIs. The blood oxygenation level-dependent (BOLD) responses were evaluated using a general linear model including regressors of interest, which were the sentence-length and paragraph-length text correlations between the text read and the content report. The text correlations were calculated identically to those in the EEG analysis, except for one parameter in Word2Vec (a context window parameter was changed from 10 to 15 words to maximize the difference between the correlations of the content report with the text read and the correlations with non-corresponding texts). In contrast to the fixation-related EEG analysis, the text correlation was associated with the BOLD signals at each scanning time, in which many eye fixations were included. To identify neural mechanisms underlying text correlations, we hypothesized an expected BOLD response by convolving the canonical hemodynamic response function with the text correlations for each participant and session. The model additionally included the time taken to press the button and six motion regressors obtained from the registration process.

Before performing the regression analysis, low-frequency confounding effects were removed using a high-pass filter with a 120-s cutoff period, and serial correlations among the scans were estimated using an autoregressive model [AR(1)] to remove the high-frequency noise contaminating the EPI time series. The parameter estimates were computed for each subject using a fixed-effects model and then taken into the group analysis using a random-effects model of a $t$ statistic (uncorrected $p<0.001$, cluster-wise FDR; $p<$ 0.05).

\section{Results}

\section{Behavioral results}

The 13 participants who underwent EEG measurements took a mean time of $7.4 \mathrm{~min}$ (SD, $2.4 \mathrm{~min}$ ) to read each of four essays and write four content reports with a mean length of 205.8 words (SD, 130.8 words) in a mean time of $12.0 \mathrm{~min}$ (SD, $6.4 \mathrm{~min}$ ). The performance (the entire text correlation between the content reports and the text read) was calculated as $0.80 \pm 0.05$ (mean \pm SD) which was significantly larger than the surrogate text correlation $\left(0.61 \pm 0.06\right.$; paired $t$ test, $\left.t_{(12)}=102.7, p<0.001\right)$. It was therefore clearly demonstrated that the participants properly described the content of the text read (Fig. 1B).

In the fMRI experiment, all 17 participants read each of the two essays during a mean period of $8.2 \mathrm{~min}$ (SD, 1.9 min; $246.4 \pm 55.8$ volumes) and wrote content reports having a mean length of 281.3 words (SD, 85.5 words) within $10 \mathrm{~min}$. The memory performance was calculated as $0.77 \pm 0.06$ (mean $\pm \mathrm{SD}$; $t$ statistic comparing the text correlation to surrogate text correlation $(0.44 \pm 0.13)$; $\left.t_{(16)}=52.5, p<0.001\right)$. The difference between the memory performance of fMRI participants and that of EEG participants was not significant $\left(t_{(28)}=1.74, p=\right.$ 0.09 ), suggesting that cognitive processes in the fMRI versus EEG participants were comparable.

\section{EEG results}

After rejecting atypical saccades (defined by atypical fixation duration or saccade size or too small separation from blinks), a mean of 481.8 fixations (SD, 259.0 fixations) was analyzed for each text and each participant. The mean duration of fixation was $287.5 \mathrm{~ms}$ (SD, $92.7 \mathrm{~ms}$ ), and the mean saccade size was 2.1 characters (SD, 4.7 characters). These values agreed with the typical values recorded during reading in English (Rayner, 1998).

Figure $2 A$ shows FRPs of the grand averaged signal. The red and blue plots show FRPs related to higher and lower regression coefficients for the sentence-length comparison, where higher and lower regression coefficients were defined by a median split of the regression coefficients. The shape of the FRPs appeared similarly to those reported during reading by Dimigen et al. (2011). In the FRP analysis, there were 65 clusters of (electrode, time)samples (60 positive and five negative) showing the sentence-length subsequent memory effect and 45 clusters of samples (19 positive and 26 negative) showing paragraph-length effects. The two positive clusters showing a sentence-length effect, in the time periods $0.10-0.21 \mathrm{~s}(p=$ 0.018 ) and 0.38-0.48 s ( $p=0.025)$, had Monte Carlo $p$ values that were $<0.025$ (Fig. $2 A$ ). As also shown in Figure $2 B$, both clusters broadly distributed over the scalp, while the former and the latter appeared in the left central area and in the frontal area, respectively. The former and latter clusters appeared to be associated with N1-P2 complex and P3 components, respectively. There were no significant clusters showing a paragraph-length effect. Topographic maps of the resulting regression coefficients averaged over time periods of either $0.1-0.2$ or $0.4-0.5 \mathrm{~s}$ from fixation onsets (Fig. $2 B$ ) showed that the sentence-length effect appeared from the frontal to central regions.

Figure $2 C$ shows fixation-related time-frequency maps for the raw (ocular-artifact uncorrected) and corrected EEG powers averaged over all electrodes. In the corrected time-frequency map, the power at the fixation onset, which is thought to reflect ocular artifact, was greatly reduced. However, the power in the low frequency band in the period of $>0.8 \mathrm{~s}$, which was influenced by eye blinks at $>1 \mathrm{~s}$, remained uncorrected. In the analysis of fixation-related time-frequency EEG power, there were 344 clusters of (electrode, time, frequency)-samples (187 positive and 137 negative) in the sentence-length com- 
A

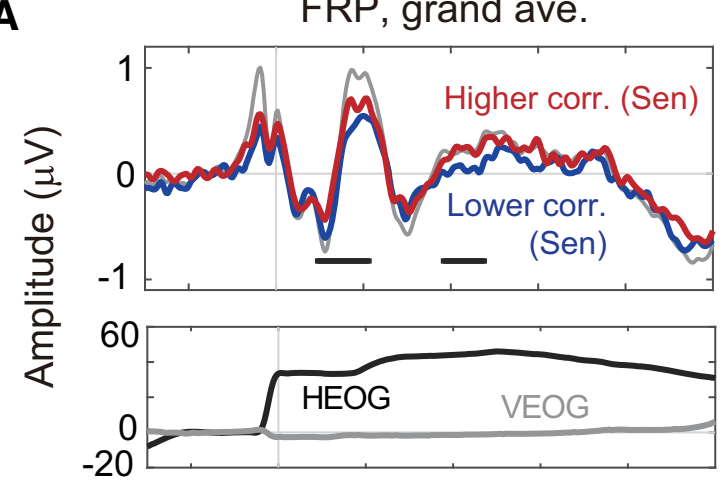

C

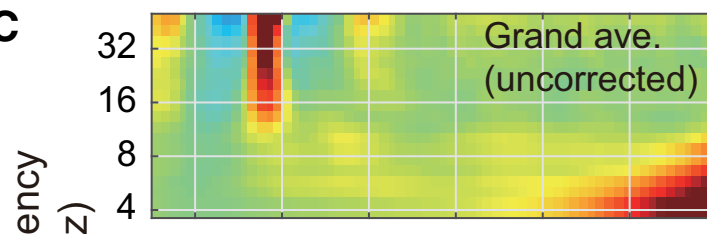

D

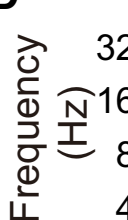

B Sentence, FRP
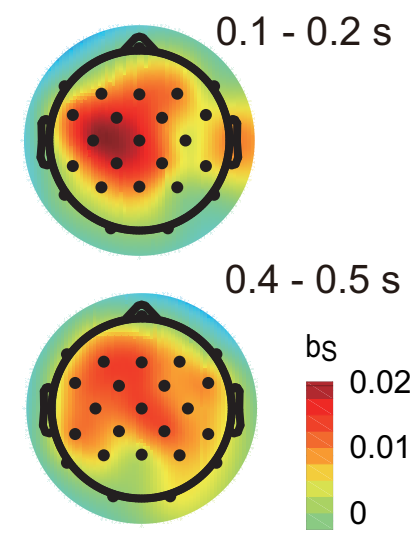

E Paragraph, theta

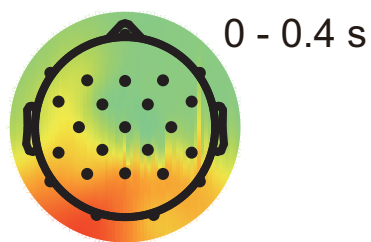

0

bP

0.02

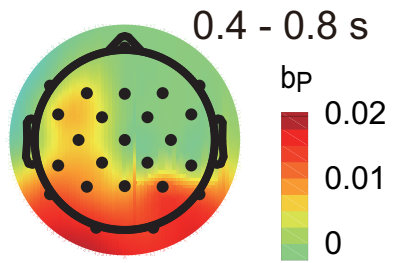

Figure 2. Results of fixation-related EEG analysis. A, FRPs of grand averaged signal over all electrodes. The upper plot shows the FRPs. The red and blue plots show FRPs during higher and lower text correlation between the read text and content reports, respectively. The "higher" and "lower" regression coefficients were defined by a median split of the regression coefficients. The gray plots show uncorrected FRP. Horizontal bars indicate time points showing significant effects $(p<0.05)$. Multiple comparisons were corrected by the nonparametric clustering permutation test (Maris and Oostenveld, 2007). The horizontal axis represents the time from fixation onset. The lower plot shows horizontal and vertical EOGs (HEOG and VEOG). B, Topographical head maps of regression coefficients of the sentence-length text correlation in the time periods $0.1-0.2$ and $0.4-0.5 \mathrm{~s}$. $\boldsymbol{C}$, Time-frequency maps of raw (ocular-artifact-uncorrected) and corrected EEG power. The horizontal axis represents the time from fixation onset; vertical axis indicates frequency on a logarithmic scale. $\boldsymbol{D}$, Time-frequency map of regression coefficients averaged over all channels for the paragraph-length text correlations between the text read and the content report. The values were masked by a cluster-based statistical value $(\boldsymbol{D}<0.05)$. $\boldsymbol{E}$, Topographical head maps of regression coefficients of the sentence-length and paragraph-length text correlations in $\theta$-band (4-8 $\mathrm{Hz}$ ) EEG. The maps of regression coefficients were averaged over the time periods $0-0.4$ and $0.4-0.8 \mathrm{~s}$.

parison and 317 clusters of samples (162 positive and 155 negative) in the paragraph-length comparison. Only one positive cluster, for the $\theta$-band in the paragraph-length comparison $(p=0.038)$, had a Monte Carlo $p$ value that was $<0.025$. There were no significant clusters in the sentence-length comparison. Figure $2 D$ shows the fixation-related time-frequency map for regression coefficients averaged over all electrodes in the paragraph-length comparison. The topographic maps of the resulting regression coefficients of $\theta$-band EEG power averaged over time periods of either $0.0-0.4$ or $0.4-0.8 \mathrm{~s}$ from fixation onsets
(Fig. 2E) showed that the paragraph-length effect appeared over the occipital region and left fronto-temporal region.

No eye movement-related parameters showed a significant correlation to subsequent memory in sentencelength or in paragraph-length comparisons (saccade size; sentence-length correlation, $t_{(12)}=1.15, p=0.27$, paragraph-length correlation, $t_{(12)}=0.47, p=0.64$, fixation duration; sentence-length correlation, $t_{(12)}=-1.93$, $p=0.08$; paragraph-length correlation, $t_{(12)}=1.96, p=$ $0.07)$. This result suggests that the significant correlation between fixation-related EEG and subsequent memory 

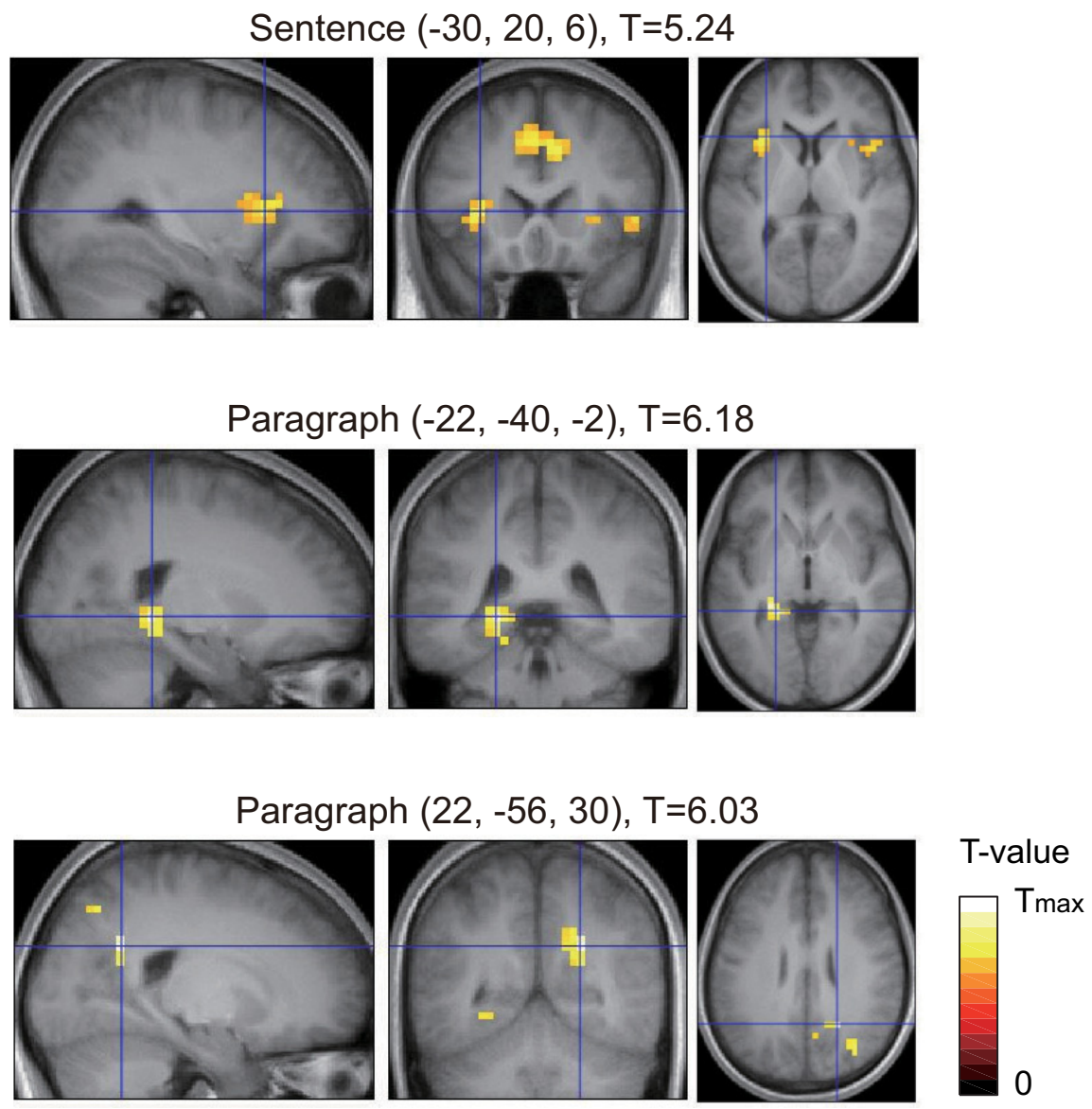

Figure 3. Brain regions showing a significant decrease in BOLD activity during reading of texts that were highly correlated with the content reports subsequently written by the participants. The results of sentence-length text correlation are shown on the top and in the middle, and those relevant to paragraph-length text correlation are shown on the bottom. There were no significant regions showing positive subsequent memory effect (i.e., BOLD activity increasing during reading of texts that were highly correlated with the content reports).

did not result from contamination by ocular artifacts. An additional analysis using three regressors (sentencelength and paragraph-length text correlations and fixation duration) yielded results that were almost identical (data not shown).

\section{fMRI results}

BOLD activities during text reading were analyzed by the time series of text correlations calculated with sentencelength and paragraph-length text comparisons between the text read and the content report. The results show no regions with a positive correlation between BOLD responses and the text correlation (i.e., no regions in which BOLD activity increased on reading the texts that were highly correlated with the content reports). In contrast, multiple regions showed significant negative correlation between the BOLD response and the text correlations (i.e., BOLD decreases during reading with high correlations between content reports and text being read; Fig. 3; Table 1). The sentence-length NSME was found in bilateral insula (BA13), right inferior frontal gyrus (BA47), and anterior cingulate gyrus (BA32). The paragraph-length NSME was observed in the left hippocampus/parahippocampal gyrus, the right pre- cuneus/posterior cingulate gyrus (BA31), and the right intraparietal sulcus (BA7).

\section{Discussion}

We found that literature reading produced a positive relationship of fixation-related EEG and negative relationship of BOLD activity to subsequent memory as measured by semantic correlation between the text read and the content reports subsequently written by the participants. The following sections discuss the potential importance of these results for the understanding of neural dynamics of memory encoding during literature reading.

\section{Positive relationship between fixation-related EEG and subsequent memory}

In the results, the sentence-length and paragraphlength effects were differently associated with FRPs and fixation-related EEG $\theta$, respectively (Fig. 2). First, the sentence-length effects were positively associated with N1-P2 and P3 components (Fig. 2A). There are no reports, to our knowledge, on the relationship between FRPs and subsequent memory, although many researchers have reported increased P3 ERP components during success- 
Table 1. Brain regions showing a significant decrease in BOLD activity correlated with sentence-length and paragraph-length text correlations

\begin{tabular}{|c|c|c|c|c|}
\hline \multirow[t]{2}{*}{ Anatomical region } & \multicolumn{3}{|c|}{ MNI coordinates (mm) } & \multirow[t]{2}{*}{$t$ value } \\
\hline & $\mathrm{x}$ & $\mathrm{y}$ & z & \\
\hline R-insula (BA13) & 50 & 0 & -10 & 7.09 \\
\hline R-cingulate gyrus (BA32) & 10 & 24 & 38 & 5.90 \\
\hline L-cingulate gyrus (BA32) & -10 & 24 & 42 & 5.22 \\
\hline L-insula (BA13) & -30 & 20 & 6 & 5.24 \\
\hline R-precuneus/cingulate gyrus (BA31) & 22 & -56 & 30 & 6.03 \\
\hline R-intraparietal sulcus (BA7) & 30 & -72 & 38 & 4.76 \\
\hline
\end{tabular}

$p<0.001$ (uncorrected) with a cluster-wise FDR of $p<0.05$.

ful encoding (Fernández et al., 1999; Klimesch et al., 2000). The current results agree with these data. On the other hand, the current results of the N1-P2 component does not simply agree with previous results; the current results appeared similar to the reading-related ERP components within 100-200 ms (mainly N1, but also the P2 component), which is thought to be associated with lexical word access (Sereno et al., 1998; Sereno and Rayner, 2003). However, topographic maps of these results, including both increases and decreases of the readingrelated ERP amplitudes in multiple regions, are not directly associated with the current data, except for the left-hemisphere dominance. In addition to FRP, N1 ERP has been extensively investigated and is thought to be associated with visual discrimination process (for review, see Luck et al., 2000). This suggests that the current result of decreased $\mathrm{N} 1$ amplitude indicates a decrease in the visual discrimination process. In summary, the interpretations of the P3 and N1-P2 components could be combined as follows. The decreased lexical access and/or decreased visual discrimination interpreted by the N1-P2 can be explained by many factors, while factors related to "low mnemonic load," rather than factors related to resting, would agree with the successful encoding interpreted by the P3. Furthermore, the low mnemonic load was speculated to be associated with "known contents" (or a good correspondence should be addressed to preexisting knowledge during reading) rather than "poor contents," because enough semantic contents in the read text were required to detect successful encoding by text correlation in the current study. It should be noted that the current results did not include either N1 FRP at the occipital region, which was shown to be associated with reading (Henderson et al., 2013), or N400 ERP (Kutas and Hillyard, 1980; Kutas and Federmeier, 2000) and N400 FRP (Dimigen et al., 2011), which are shown to be associated with text comprehension.

Second, the paragraph-length effect was associated with increased EEG $\theta$ (Fig. 2D,E). There are a number of reports showing increased EEG $\theta$ during successful encoding (Klimesch et al., 1996; Weiss and Rappelsberger, 2000; Sederberg et al., 2003; Summerfield and Mangels, 2005; Osipova et al., 2006). The current results agree with these data, except for the topographic maps of the SME; the current results appeared in fronto-temporal and occipital regions, while previous reports differently appeared in frontal (Summerfield and Mangels, 2005; White et al., 2013), central (Klimesch et al., 2001), or temporal regions (Hanslmayr et al., 2011; for review, see Hsieh and Ranganath, 2014). Besides the successful encoding, the current results could be also associated with a more extensive mnemonic processes, such as linguistic comprehension associated with left fronto-temporal $\theta$ increases (Hagoort et al., 2004), or a higher load of cognitive control associated with left fronto-temporal $\theta$ increases (Sauseng et al., 2010).

\section{Negative relationship between BOLD activity and subsequent memory}

Increases (Brewer, 1998; Wagner, 1998) as well as decreases (Otten and Rugg, 2001; Wagner and Davachi, 2001; Daselaar et al., 2004; de Chastelaine and Rugg, 2014) in BOLD activity have both been reported during successful encoding (for review, see Kim, 2011). Our results failed to identify regions showing positive relationships between BOLD signals and subsequent memory, while multiple regions showed negative correlations with subsequent memory (i.e., NSME), with the bilateral insula, right inferior frontal gyrus and anterior cingulate gyrus showing the sentence-length effect and the left hippocampus/parahippocampal gyrus, the right precuneus/ cingulate gyrus, and the right inferior frontal gyrus showing the paragraph-length effect (Fig. 3; Table 1).

For the interpretation of the current results, the overlaps of the resultant regions with SME/NSME regions and other functional networks in previous reports were computed (Table 2). Surprisingly, the current results were found to be not well correlated with the regions showing NSME in a previous report (Kim, 2011), suggesting that the regions showing negative relationships to subsequent memory do not simply reflect successful memory encoding. The regions showing a sentence-length effect are primarily associated with the anterior salience network (Menon and Uddin, 2010), which functions to identify the most relevant among several internal and extra-personal stimuli to guide behavior. In contrast, the regions showing a paragraph-length effect largely overlapped with the default mode network (DMN; Raichle et al., 2001), which is 
Table 2. Volume overlap ratio of the NSME regions in this study with previously reported functional networks

\begin{tabular}{|c|c|c|c|c|c|c|c|c|}
\hline \multirow[b]{2}{*}{ Anatomical region } & \multicolumn{2}{|c|}{$\begin{array}{l}\text { SME/NSME networks } \\
\text { (Kim, 2011) }\end{array}$} & \multicolumn{6}{|c|}{$\begin{array}{l}\text { Functional networks } \\
\text { (Shirer et al., 2012) }\end{array}$} \\
\hline & SME & NSME & ASN & dDMN & vDMN & PN & LECN & VSN \\
\hline Cingulate gyrus & & & $0.92^{*}$ & 0.15 & & & 0.03 & \\
\hline L-insula & & & $0.40^{*}$ & & & & & \\
\hline $\begin{array}{l}\text { R-insula/inferior frontal gyrus } \\
\text { Paragraph-length NSME }\end{array}$ & $0.41^{*}$ & & $0.37^{*}$ & & & & & 0.05 \\
\hline R-precuneus/cingulate gyrus & & $0.18^{*}$ & & $0.41^{*}$ & 0.20 & $0.37^{*}$ & & 0.04 \\
\hline L-hippocampus/parahippocampal gyrus & & & & 0.01 & 0.04 & & & \\
\hline R-intraparietal sulcus & & & & & $0.35^{*}$ & 0.11 & $0.51^{*}$ & $0.37^{*}$ \\
\hline
\end{tabular}

SME/NSME networks were defined by multiple spheres of which locations and volumes were given by SME (verbal associative subgroup) and NSME (verbal item subgroup; Table 3 and 6 in Kim, 2011, respectively). Functional ROls reported by Shirer et al. (2012) were analyzed. 6/14 functional networks showing significant overlap are listed in the table. Volume overlap was defined by (overlapped voxels)/(\#voxels in the ROI). Each functional network was inflated \pm 1 voxel $(3 \mathrm{~mm})$ to give stable overlap. * indicates significance at the level of $p<0.05$ with FDR correction $(q<0.05)$, with a null hypothesis of "volume overlap ratio between ROls and functional regions is equal to the volume ratio of the region to the domain (voxels included in every network)". ASN: anterior salience network; dDMN and vDMN: dorsal and ventral DMNs; PN: precuneus network; LECN: left-executive control network; VSN: visuospatial network.

known to be activated during relaxed non-task states and self-oriented cognition, such as mind wandering or autobiographical memory retrieval, and is deactivated during performing cognitive tasks (for review, see Buckner et al., 2008; Raichle, 2015).

The result of sentence-length NSME in the salience network could superficially produce a contradictive interpretation, i.e., successful encoding was associated with decreased attention to the text. However, this can be solved by considering the details of the decreased attention as follows. The salience network was thought to be continuously activated during reading, while its activity is supposed to be relatively decreased during the reading of texts characterized by low mnemonic load, such as "known texts," as illustrated by the EEG sentence-length effect. Cognitive factors related to resting, e.g., low wakefulness or low interest to the text, were also associated with decreased attention to the text. However, these effects, of which time course was supposed to be longer than the time course of individual sentence reading, were expected to be regressed out from the sentence-length effects in the current multiple regressions analysis, and that would be dominant in the paragraph-length effect. The texts characterized by low mnemonic load were supposed to be easily memorized, thus the sentence-length NSME in the salience network is explained

The result of paragraph-length NSME in the DMN was explained by the suppression of task-irrelevant internal thoughts (Anticevic et al., 2012) and the effective allocation of cortical resources (Hasson et al., 2007). In contrast to the salience network, the DMN was thought to be continuously deactivated during reading, while the DMN could be more strongly deactivated during higher efforts of reading when task-irrelevant internal thoughts were more strongly suppressed. During such periods, the encoding performance was also supposed to be better. As a result, the paragraph-length NSME in the DMN would appear. As discussed above, with the consideration of the time course of the task-irrelevant internal thoughts, this effect would likely appear as a paragraph-length effect, rather than a sentence-length effect.

The results of NSMEs in both the salience network and the DMN seemingly contradict that these networks are usually known to have opposite activation patterns. However, this is simply solved by considering the differences of the time courses of the detected BOLD signals in these networks. In the current essays used, the average paragraph-length was two-and-a-half-times longer than the average sentence-length (See EEG Methods/Stimuli); accordingly, the time course of the paragraph-length regressors was sufficiently longer than the time course of the sentence-length regressor. Thus, it was thought that the NSMEs in the salience network and the DMN were not contradictory, but instead reflected differences in cognitive aspects in different time courses; the former reflected the reading of individual sentences characterized by low mnemonic load and the latter reflected the cognitive states related to the suppression of task-irreverent thoughts.

The hippocampus usually showed a positive SME, but our results showed an NSME for the left hippocampus/ parahippocampal gyrus. This failure to detect increased BOLD activity during successful encoding may be explained by the long-lasting reading $(\sim 10 \mathrm{~min})$ in the current task, in which the regions associated with subsequent memory were continuously activated. A relatively small fluctuation dependent on subsequent memory may be obscured by a more general pattern of task-related deactivation, as pointed out by Yarkoni et al. (2008). Recently, Baldassano et al. (2017) demonstrated that the hippocampus was specifically activated at the end of an event, while the average hippocampal activity was decreased during the event. This may also explain the current result.

\section{Relationship between fixation-related EEG and BOLD activity}

Simultaneous fMRI-EEG measurements have demonstrated an inverse correlation between frontal EEG $\theta$ and BOLD activity in the DMN during resting (Scheeringa et al., 2008), mental arithmetic (Mizuhara et al., 2004), and memory encoding (Sato et al., 2010; White et al., 2013). The current results for the paragraph-length comparison showed a negative relationship with fronto-temporal EEG $\theta$ and a positive relationship with BOLD activity in the $D M N$, which is in agreement with these previous reports. 
On the other hand, combined ERP-fMRI studies have demonstrated a positive correlation between P3a components (an earlier subcomponent of P3) and BOLD activities in frontal areas and the insula during oddball tasks (Bledowski et al., 2004), and in the anterior cingulate during spatial attention tasks (Bengson et al., 2015). The current results of the sentence-length comparison, showing a positive relationship with N1-P2 and P3 FRP components and a negative relationship with BOLD activity in the salience network, does not agree with these previous reports. This might be explained by differences in the cognitive tasks; the current task of natural reading required more complicated cognitive processes than the tasks described in the previous reports. Another reason is thought that ERPs originating from phase resetting might not induce major changes in local brain metabolism, as pointed out by Debener et al. (2006).

\section{Text correlation as an index of subsequent memory}

There are different levels of text comprehension; textbase comprehension is the encoding of the meaning of the text and situation model construction is further supplemented by preexisting knowledge needed for coherent understanding (Kintsch, 1994). Free-recall tasks have been typically used for the evaluation of textbase comprehension; however, the text correlation used in the current study was thought to measure subsequent memory associated with both the textbase and situation model. One reason for this was that the text read was long enough ( $~ 8$ and 4 thousand words, i.e., $\sim 30$ and 15 min of reading, in EEG and $\mathrm{fMRI}$ experiments, respectively) to allow memorization of phrases in the text; however, semantic context and preexisting knowledge were still available during reading as effective guides for encoding. This may lead participants to use a strategy that falls within the situation model for the encoding of text. This was partially supported by the results of content reports, each of which consisted of a small number of words $(\sim 10 \%$ of the text read), but included abstract representations of the text read. On the other hand, the possible use of preexisting knowledge during reading may produce a problematic variation in encoding strategies. Unfortunately, the current study cannot rule out this possibility; however, behavioral results showing no explicit outliers in reading time and the word number of content reports suggests a relatively small variation in participant encoding strategies.

In summary, our results demonstrated (1) the availability of semantic correlation of text, based on a recent natural language processing procedure, to detect brain activities related to context-dependent memory; (2) the positive relationship of FRP and fixation-related EEG $\theta$ and negative relationship of BOLD activity with subsequent memory; and (3) the different time courses of memory-related activities in the salience network and the DMN during reading, in which the sentence-length encoding was associated with salience network deactivation (thought to reflect the reading of sentences characterized by low mnemonic load), and the paragraph-length encoding was associated with the DMN deactivation (thought to reflect the suppression of task-irreverent thoughts during read- ing). It has been suggested that context-dependent memory encoding during natural reading of literature requires large-scale network deactivation that might reflect the coordination of a range of voluntary processes during reading.

\section{References}

Anticevic A, Cole MW, Murray JD, Corlett PR, Wang XJ, Krystal JH (2012) The role of default network deactivation in cognition and disease. Trends Cogn Sci 16:584-592. CrossRef Medline

Baldassano C, Chen J, Zadbood A, Pillow JW, Hasson U, Norman KA (2017) Discovering event structure in continuous narrative perception and memory. Neuron 95:709-721.e5. CrossRef

Bastiaansen M, Hagoort P (2006) Oscillatory neuronal dynamics during language comprehension. Progr Brain Res 159:179-196. CrossRef Medline

Bengson JJ, Kelley TA, Mangun GR (2015) The neural correlates of volitional attention: a combined fMRI and ERP study. Hum Brain Mapp 36:2443-2454. CrossRef Medline

Bledowski C, Prvulovic D, Hoechstetter K, Scherg M, Wibral M, Goebel R, Linden DE (2004) Localizing P300 generators in visual target and distractor processing: a combined event-related potential and functional magnetic resonance imaging study. J Neurosci 24:9353-9360. CrossRef

Blei DM, Ng AY, Jordan MI (2003) Latent Dirichlet allocation. J Mach Learn Res 3:993-1022.

Brennan J (2016) Naturalistic sentence comprehension in the brain. Lang Linguist Compass 10:299-313. CrossRef

Brewer JB (1998) Making memories: brain activity that predicts how well visual experience will be remembered. Science 281:11851187. CrossRef

Buckner RL, Andrews-Hanna JR, Schacter DL (2008) The brain's default network: anatomy, function, and relevance to disease. Ann NY Acad Sci 1124:1-38. CrossRef Medline

Cutting LE, Clements AM, Courtney S, Rimrodt SL, Schafer JG, Bisesi J, Pekar JJ, Pugh KR (2006) Differential components of sentence comprehension: beyond single word reading and memory. Neuroimage 29:429-438. CrossRef Medline

Daselaar SM, Prince SE, Cabeza R (2004) When less means more: deactivations during encoding that predict subsequent memory. Neuroimage 23:921-927. CrossRef Medline

de Chastelaine M, Rugg MD (2014) The relationship between taskrelated and subsequent memory effects. Hum Brain Mapp 35: 3687-3700. CrossRef Medline

Debener S, Ullsperger M, Siegel M, Engel AK (2006) Single-trial EEG-fMRI reveals the dynamics of cognitive function. Trends Cogn Sci 10:558-563. CrossRef Medline

Dimigen O, Sommer W, Hohlfeld A, Jacobs AM, Kliegl R (2011) Coregistration of eye movements and EEG in natural reading: analyses and review. J Exp Psychol Gen 140:552-572. CrossRef Medline

Fernández G, Effern A, Grunwald T, Pezer N, Lehnertz K, Dümpelmann M, Van Roost D, Elger CE (1999) Real-time tracking of memory formation in the human rhinal cortex and hippocampus. Science 285:1582-1585. Medline

Ferstl EC, von Cramon DY (2001) The role of coherence and cohesion in text comprehension: an event-related fMRI study. Brain Res Cogn Brain Res 11:325-340. Medline

Hagoort P, Hald L, Bastiaansen M, Petersson KM (2004) Integration of word meaning and world knowledge in language comprehension. Science 304:438-441. CrossRef Medline

Hanslmayr S, Staudigl T (2014) How brain oscillations form memories-a processing based perspective on oscillatory subsequent memory effects. Neuroimage 85:648-655. CrossRef

Hanslmayr S, Volberg G, Wimber M, Raabe M, Greenlee MW, Bäuml $\mathrm{KH}$ (2011) The relationship between brain oscillations and BOLD signal during memory formation: a combined EEG-fMRI study. J Neurosci 31:15674-15680. CrossRef Medline 
Hasson U, Nusbaum HC, Small SL (2007) Brain networks subserving the extraction of sentence information and its encoding to memory. Cereb Cortex 17:2899-2913. CrossRef Medline

Henderson JM, Luke SG, Schmidt J, Richards JE (2013) Coregistration of eye movements and event-related potentials in connected-text paragraph reading. Front Syst Neurosci 7:28. CrossRef Medline

Hsieh LT, Ranganath C (2014) Frontal midline theta oscillations during working memory maintenance and episodic encoding and retrieval. Neuroimage 85:721-729. CrossRef

Huth AG, Nishimoto S, Vu AT, Gallant JL (2012) A continuous semantic space describes the representation of thousands of object and action categories across the human brain. Neuron 76:12101224. CrossRef Medline

Hyvärinen A, Oja E (2000) Independent component analysis: algorithms and applications. Neural Netw 13:411-430. Medline

Kim H (2011) Neural activity that predicts subsequent memory and forgetting: a meta-analysis of $74 \mathrm{fMRI}$ studies. Neuroimage 54: 2446-2461. CrossRef Medline

Kintsch W (1994) Text comprehension, memory, and learning. Am Psychol 49:294-303. Medline

Klimesch W, Doppelmayr M, Russegger H, Pachinger T (1996) Theta band power in the human scalp EEG and the encoding of new information. Neuroreport 7:1235-1240. Medline

Klimesch W, Doppelmayr M, Schwaiger J, Winkler T, Gruber W (2000) Theta oscillations and the ERP old/new effect: independent phenomena? Clin Neurophysiol 111:781-793. CrossRef

Klimesch W, Doppelmayr M, Stadler W, Pöllhuber D, Sauseng P, Röhm D (2001) Episodic retrieval is reflected by a process specific increase in human electroencephalographic theta activity. Neurosci Lett 302:49-52. Medline

Kutas M, Hillyard SA (1980) Reading senseless sentences: brain potentials reflect semantic incongruity. Science 207:203-205. Medline

Kutas M, Federmeier KD (2000) Electrophysiology reveals semantic memory use in language comprehension. Trends Cogn Sci 4:463470. Medline

Landauer TK, Dumais ST (1997) A solution to Plato's problem: the latent semantic analysis theory of acquisition, induction, and representation of knowledge. Psychol Rev 104:211-240. CrossRef

Luck SJ, Woodman GF, Vogel EK (2000) Event-related potential studies of attention. Trends Cogn Sci 4:432-440. Medline

Maekawa K, Yamazaki M, Ogiso T, Maruyama T, Ogura H, Kashino W, Koiso H, Yamaguchi M, Tanaka M, Den Y (2014) Balanced corpus of contemporary written Japanese. Lang Resour Eval 48: 345-371. CrossRef

Maris E, Oostenveld R (2007) Nonparametric statistical testing of EEG- and MEG-data. J Neurosci Methods 164:177-190. CrossRef Medline

Menon V, Uddin LQ (2010) Saliency, switching, attention and control: a network model of insula function. Brain Struct Funct 214:655667. CrossRef Medline

Mikolov T, Chen K, Corrado G, Dean J (2013) Efficient estimation of word representations in vector space. arXiv preprint arXiv 1301.3781.

Mitchell TM, Shinkareva SV, Carlson A, Chang KM, Malave VL, Mason RA, Just MA (2008) Predicting human brain activity associated with the meanings of nouns. Science 320:1191-1195. CrossRef Medline

Mizuhara H, Wang L-Q, Kobayashi K, Yamaguchi Y (2004) A longrange cortical network emerging with theta oscillation in a mental task. Neuroreport 15:1233-1238. CrossRef

Nyhus E, Curran T (2010) Functional role of gamma and theta oscillations in episodic memory. Neurosci Biobehav Rev 34:10231035. CrossRef Medline

Osipova D, Takashima A, Oostenveld R, Fernández G, Maris E, Jensen O (2006) Theta and gamma oscillations predict encoding and retrieval of declarative memory. J Neurosci 26:7523-7531. CrossRef Medline
Otten LJ, Rugg MD (2001) When more means less: neural activity related to unsuccessful memory encoding. Curr Biol 11:15281530. Medline

Pallier C, Devauchelle A-D, Dehaene S (2011) Cortical representation of the constituent structure of sentences. Proc Natl Acad Sci USA 108:2522-2527. CrossRef

Raichle ME (2015) The brain's default mode network. Annu Rev Neurosci 38:433-447. CrossRef Medline

Raichle ME, MacLeod AM, Snyder AZ, Powers WJ, Gusnard DA, Shulman GL (2001) A default mode of brain function. Proc Natl Acad Sci USA 98:676-682. CrossRef Medline

Rayner K (1998) Eye movements in reading and information processing: 20 years of research. Psychol Bull 124:372-422. Medline

Sato S, Matsuyoshi S, Kondoh Y (2008) Automatic assessment of Japanese text readability based on a textbook corpus. Proceedings of the International Conference on Language Resources and Evaluation, LREC 2008.

Sato N, Ozaki TJ, Someya Y, Anami K, Ogawa S, Mizuhara H, Yamaguchi $Y$ (2010) Subsequent memory-dependent EEG theta correlates to parahippocampal blood oxygenation leveldependent response. Neuroreport 21:168-172. CrossRef Medline

Sato N (2015) Predictability of subsequent retrieval after natural reading of literature: A scalp electroencephalogram study. Program No.171.23. 2015 Neuroscience Meeting Planner. Chicago: Society for Neuroscience.

Sauseng P, Griesmayr B, Freunberger R, Klimesch W (2010) Control mechanisms in working memory: a possible function of EEG theta oscillations. Neurosci Biobehav Rev 34:1015-1022. CrossRef Medline

Scheeringa R, Bastiaansen MC, Petersson KM, Oostenveld R, Norris DG, Hagoort P (2008) Frontal theta EEG activity correlates negatively with the default mode network in resting state. Int J Psychophysiol 67:242-251. CrossRef

Sederberg PB, Kahana MJ, Howard MW, Donner EJ, Madsen JR (2003) Theta and gamma oscillations during encoding predict subsequent recall. J Neurosci 23:10809-10814. Medline

Sereno SC, Rayner K (2003) Measuring word recognition in reading: eye movements and event-related potentials. Trends Cogn Sci 7:489-493. Medline

Sereno SC, Rayner K, Posner MI (1998) Establishing a time-line of word recognition: evidence from eye movements and event-related potentials. Neuroreport 9:2195-2200. Medline

Shirer WR, Ryali S, Rykhlevskaia E, Menon V, Greicius MD (2012) Decoding subject-driven cognitive states with whole-brain connectivity patterns. Cereb Cortex 22:158-165. CrossRef

Summerfield C, Mangels JA (2005) Coherent theta-band EEG activity predicts item-context binding during encoding. Neuroimage 24: 692-703. CrossRef Medline

Wagner AD (1998) Building memories: remembering and forgetting of verbal experiences as predicted by brain activity. Science 281 : 1188-1191. Medline

Wagner AD, Davachi L (2001) Cognitive neuroscience: forgetting of things past. Curr Biol 11:R964-R967. Medline

Weiss S, Rappelsberger P (2000) Long-range EEG synchronization during word encoding correlates with successful memory performance. Brain Res Cognitive Brain Res 9:299-312. Medline

White TP, Jansen M, Doege K, Mullinger KJ, Park SB, Liddle EB, Gowland PA, Francis ST, Bowtell R, Liddle PF (2013) Theta power during encoding predicts subsequent-memory performance and default mode network deactivation. Hum Brain Mapp 34:2929 2943. CrossRef Medline

Woldorff MG (1993) Distortion of ERP averages due to overlap from temporally adjacent ERPs: analysis and correction. Psychophysiology 30:98-119. Medline

Xu J, Kemeny S, Park G, Frattali C, Braun A (2005) Language in context: emergent features of word, sentence, and narrative comprehension. Neuroimage 25:1002-1015. CrossRef Medline

Yarkoni T, Speer NK, Zacks JM (2008) Neural substrates of narrative comprehension and memory. Neuroimage 41:1408-1425. CrossRef Medline 